\title{
SEM contrast of solid surfaces irradiated with highly charged ions
}

\author{
Makoto Sakurai, Hiroyuki Onishi, Ken Asakura, Naoyuki Iida, Masahide \\ Tona*, Toshifumi Terui**, and Shinro Mashiko** \\ Department of Physics, Kobe University, 1-1 Rokkodai, Nada, Kobe 657-8501, Japan \\ Fax: 81-78-803-5648, e-mail: msakurai@kobe-u.ac.jp \\ *Department of Chemistry, Kobe University, 1-1 Rokkodai, Nada, Kobe 657-8501, Japan \\ Fax: 81-78-803-5770, e-mail: tona@tiger.kobe-u.ac.jp \\ **National Institute of Information and Communications Technology, Iwaoka, Kobe 651-2492, Japan \\ Fax: 81-78-969-2259, e-mail: terui@nict.go.jp
}

\begin{abstract}
We have observed secondary electron microscope (SEM) images of the surfaces of Si with native oxide layers and highly oriented pyrolytic graphite (HOPG) irradiated with highly charged ions (HCIs, Ar ${ }^{11+}$ ) produced by an

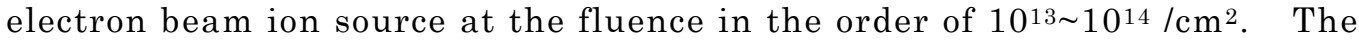
contrast of SEM image changes brighter at irradiated areas for graphite surfaces, while the contrast for the Si surface become darken at irradiated areas. The SEM contrast between irradiated and unirradiated areas is understood in terms of the voltage contrast mechanism in the interpretation of SEM images. When the strength of the contrasts is roughly compared with the sample irradiated with singly charged ions (SCIs), HCI has 100 times higher efficiency for the production of SEM contrast than SCI. The high efficiency reflects the nature of interaction between HCI and surface. Key words: Highly Charged Ion, Graphite, Silicon, Secondary Electron Microscope
\end{abstract}

\section{INTRODUCTION}

The interaction of slow highly charged ions (HCIs) with surfaces has been investigated over various aspects; such as surface modification, photon and electron emission, and secondary ion emission mediated by potential sputtering mechanism [1]. The interaction is characterized by the large potential energy of HCI defined as summation of ionization energies for each ionizing step to reach specific charge state, and also by the dynamics of charge transfer process where many electrons within surface region of the target move to HCI approaching the surface in very short time $(\sim 100 \mathrm{fs})$. The surface structures have been observed for various samples irradiated with HCIs by using scanning tunneling microscope (STM). For some materials such as mica and graphite, the impact site of HCI makes protrusion in the scale of $\mathrm{nm}[2,3]$. On the other hand, for most semiconductor and insulator materials, crater-like structure is produced[4,5]. The size of the both types of structures functionally changes with the charge state of impinging $\mathrm{HCI}$.
Since the impact of single HCI on a surface produces hundreds of secondary electrons and a nanometer structure, which leads to single ion implantation technology[6,7], and impact sites get activity that specific molecules stick to the sites whereas unirradiated regions do not have such activity[8], HCI is suitable for a processing method in the production of nanometer scale devices. As a first step of application of HCI to 'nanoprocess', we have tried to make ordered array of impact regions with a size of $\mathrm{nm} \sim \mathrm{m}$ scale. It is necessary to observe the created structures for the characterization, however, STM is not suitable method because the observable area is limited less than several $\mathrm{mm}$. Secondary electron microscope (SEM) has advantageous characteristics for this purpose; high resolution $(\sim 10 \mathrm{~nm})$, variable magnification range viewing $\mathrm{m} \sim \mathrm{mm}$ area, and simple operation. In the present paper, we observed SEM images of the surfaces irradiated with HCIs in order to show that SEM is useful for discriminating irradiated areas from unirradiated ones, and the contrasts of the SEM images between 
irradiated and unirradiated areas are discussed in terms of the voltage contrast mechanism.

\section{EXPERIMENTAL}

The Ar HCIs were produced with an electron beam ion source (EBIS) at the Kobe University. The design and performance of the EBIS is described elsewhere [9,10]. The electron beam $(16 \mathrm{keV}, 80 \mathrm{~mA})$ was compressed in the drift tube of the EBIS by $3 \mathrm{~T}$ magnetic field generated by a superconducting magnet cooled by a closed-cycle refrigerator. Gaseous Ar is introduced in the drift tube and Ar HCIs are produced by successive ionization processes of high current-density electron beam. The HCIs were extracted from the EBIS at an acceleration potential of $3 \mathrm{kV}$. The beam current of extracted HCI is in the order of $1 \mathrm{nA}$.

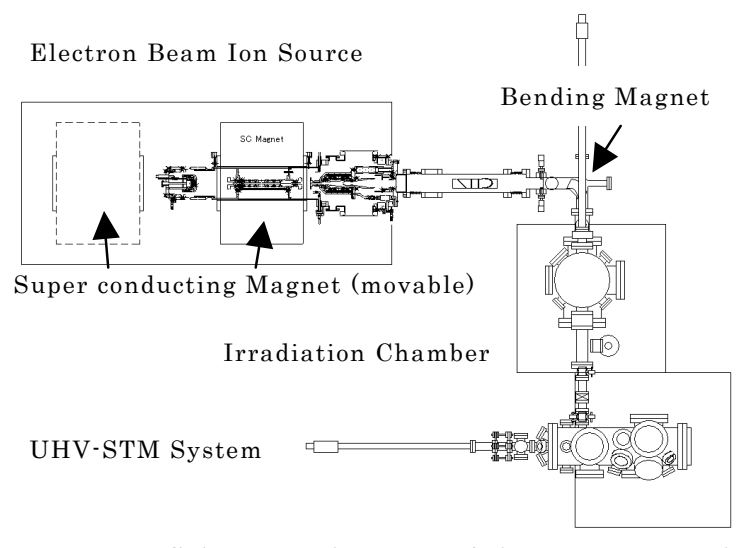

Fig. 1 Schematic diagram of the experimental apparatus.

Figure 1 shows the experimental setup, that is construction of the beamline which comprises the EBIS, a bending magnet and ion optics, an irradiation chamber and an UHV-STM system. The whole beamline is kept under UHV condition throughout the entire experimental procedures. The HCI beam was charge-separated and focused on the sample by the analyzing magnet and electrostatic lens systems located before and behind the magnet. The beam intensity is estimated at the order of $10^{10} \mathrm{~cm}^{2}$ taking into account of the beam current and beam size measured by using a Faraday cup and MCP monitor with fluorescent screen located behind the sample. The irradiation chamber is equipped with a goniometer with which the position of a base plate that supports a sample and mask for collimation of HCI beam is adjustable with respect to the incident HCI beam with degrees of freedom of XYZ translation and rotation around Z-axis. The goniometer also equips an XY stage driven by piezoelectric motors (Nanomotion Ltd.) for precise translation of the mask against the sample with the resolution of $50-100 \mathrm{~nm}$. The sample can be transferred to either the UHV-STM system or a load-lock chamber attached to the irradiation chamber.

We used a polished Si(111) wafer with native oxide layers and highly oriented pyrolytic graphite (HOPG). Any cleaning procedure was not performed for the surface of Si sample. For HOPG, topmost layers were peeled off using adhesive tape before installation to the load-lock chamber. Each sample was covered with a commercial $\mathrm{Cu}$ mesh (\#200) in contact with the sample surface in order to help comparing SEM contrasts between irradiated and unirradiated areas. The samples were irradiated with $\mathrm{Ar}^{11+}$ ions for various times ranging from 1 to 40 hours. The surfaces of irradiated samples were observed by a field emission SEM (JEOL JSM-6700F) at the Kobe Advanced ICT Research Center (KARC), National Institute of Information and Communication Technology. The irradiated samples were carried to KARC from Kobe University in atmosphere. For comparison, we also observed the SEM images of HOPG samples irradiated with singly charged ions (SCIs) of Ar produced by an ion-sputter gun (ULVAC, USG-3) attached to the STM chamber. Beam current of the gun was in the order of $0.1 \sim$ $1 \mathrm{~A}$ at the operating Ar pressure of $10^{-4} \sim$ $10^{-3} \mathrm{~Pa}$ in the STM chamber.

\section{RESULTS AND DISCUSSION}

Some SEM images of HOPG sample irradiated with $\mathrm{Ar}^{11+} \mathrm{HCIs}$ are shown in Fig. 2. The fluence of HCI irradiation is in the order of $10^{14} / \mathrm{cm}^{2}$. The incident electron energy is $1.0 \mathrm{keV}$ for Fig. 2(a), and $0.5 \mathrm{keV}$ for Fig. 2(b), respectively. Mesh patterns are observable for both images, and the contrast between irradiated and unirradiated areas is stronger for Fig. 2(b). We tried to observe similar images for the incident electron energy of $5.0 \mathrm{keV}$, however, the contrast was very weak. The contrast of SEM image changes brighter at irradiated regions (inside squares in Fig. 2). 

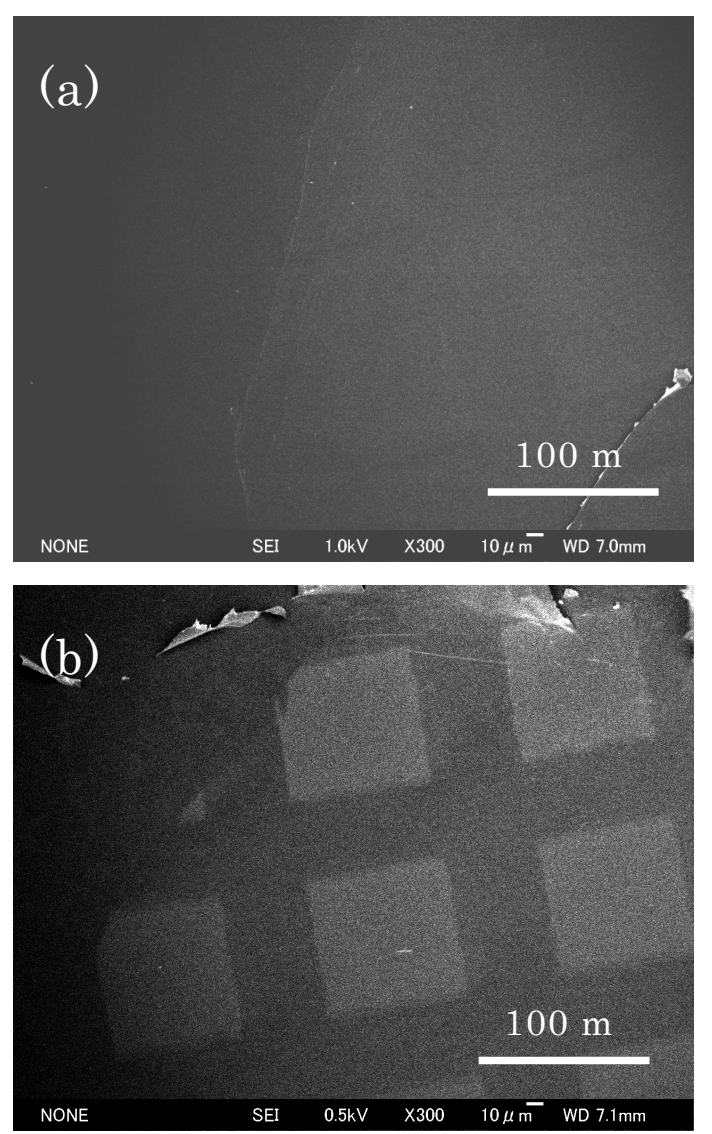

Fig. 2 SEM images of HOPG irradiated with $\mathrm{Ar}^{11+}$ HCIs. The incident electron energy for each image is $1.0 \mathrm{keV}$ for (a), and $0.5 \mathrm{keV}$ for (b), respectively. The fluence is in the order of $10^{14} / \mathrm{cm}^{2}$

Perfectness of crystal structure is deteriorated by the irradiation, and conductivity will become lower. The observed contrast is along voltage contrast mechanism in the interpretation of SEM image, however, channeling contrast mechanism might also work in this case. At the irradiated areas, primary electron scatters more frequently than that at unirradiated area, so the efficiency of secondary electron emission becomes higher and the image becomes brighter. The mesh pattern was not visible with an optical microscope.

Fig. 3 shows the SEM images of HOPG irradiated with singly charged Ar ions. The fluence of ion irradiation is in the order of $10^{17 / \mathrm{cm}^{2}}$. The incident electron energy for each image is $5.0 \mathrm{keV}$ for Fig. 3(a), $1.0 \mathrm{keV}$ for Fig. 3(b), and $0.5 \mathrm{keV}$ for Fig. 3(c), respectively. Darker regions at the center of the images correspond to areas shadowed by circular frame of the mesh, that is
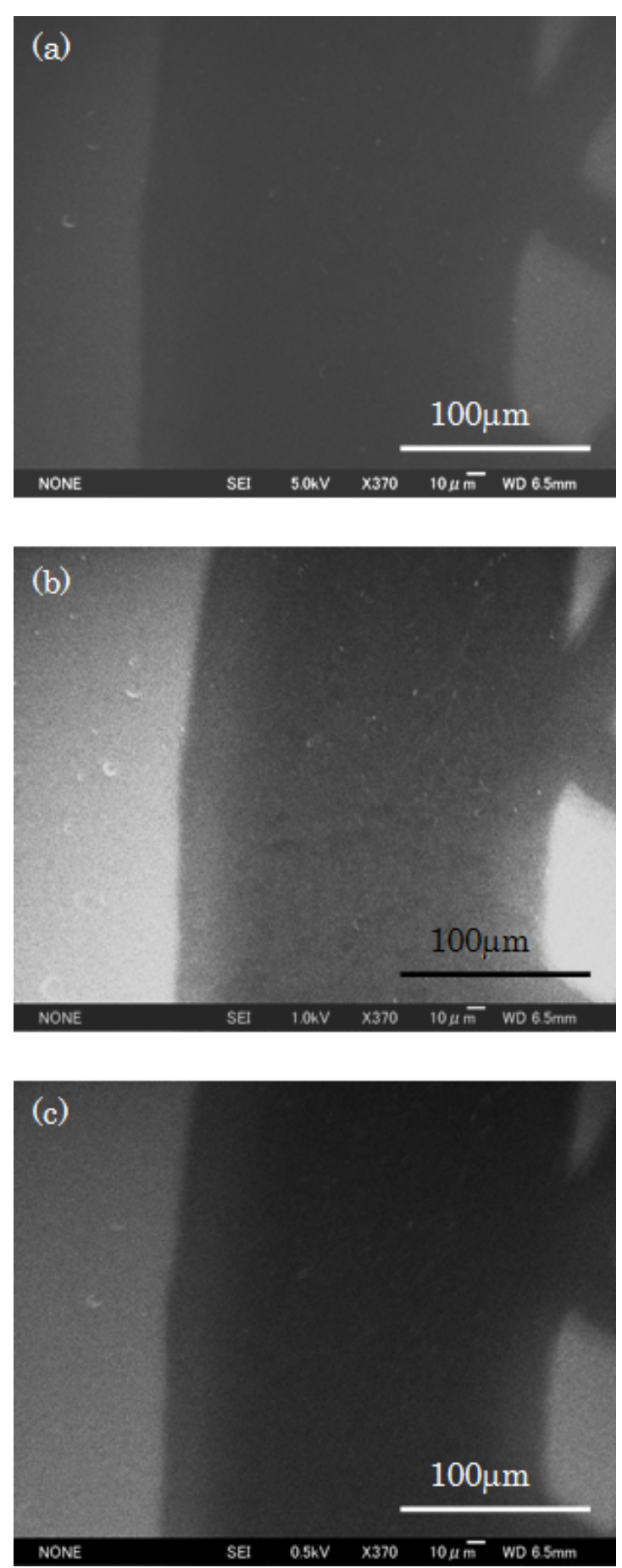

Fig. 3. SEM images of HOPG irradiated with $\mathrm{Ar}^{+}$ions. The incident electron energy for each image is $5.0 \mathrm{keV}$ for (a), $1.0 \mathrm{keV}$ for (b), and $0.5 \mathrm{keV}$ for (c), respectively. The fluence is in the order of $10^{17} / \mathrm{cm}^{2}$.

unirradiated areas. The contrast does not change remarkably with the incident electron energy. The difference in the incident energy dependences of SEM images between HCI irradiated samples and SCI irradiated ones reflects the characteristics 
of ion-surface interaction.

The potential energy of HCI $(\sim 2 \mathrm{keV}$ for $\left.\mathrm{Ar}^{11+}\right)$ is dissipated at a few atomic layers $(\sim 0.3 \mathrm{~nm})$ at the surface, while kinetic energy $(33 \mathrm{keV})$ is distributed over many layers $(\sim 10 \mathrm{~nm})$ near the surface region.
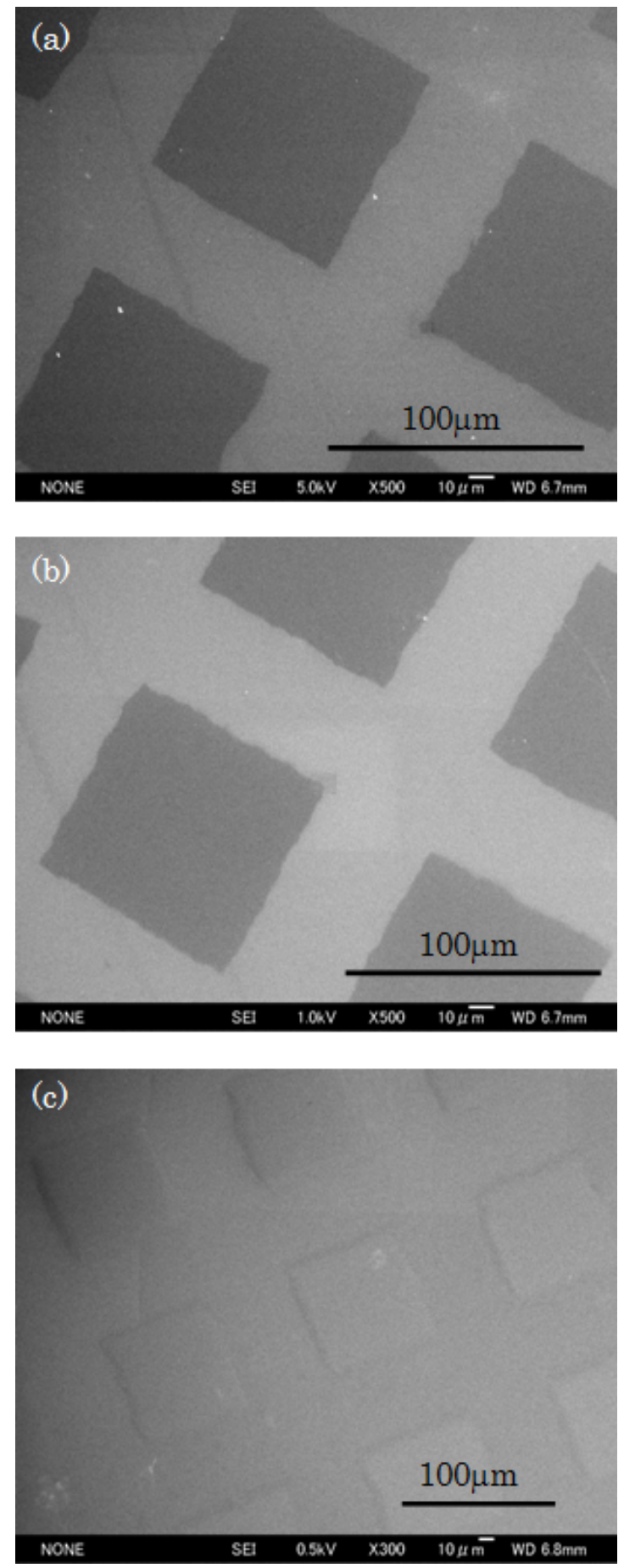

Fig. 4 SEM images of Si irradiated with Ar ${ }^{11+}$ HCIs. The incident electron energy for each image is $5.0 \mathrm{keV}$ for (a), $1.0 \mathrm{keV}$ for (b), and $0.5 \mathrm{keV}$ for (c), respectively. The fluence is in the order of $10^{14} / \mathrm{cm}^{2}$.
This means that the efficiency of surface modification is much higher for the potential energy effect compared to the kinetic energy effect. Since the SEM contrast depends on the mean free path of both incident and secondary electrons, and the mean free path of incident electron is shortest at $0.5 \mathrm{keV}(\sim 10 \mathrm{~nm})$ among the present conditions, the present results means the SEM image at $0.5 \mathrm{keV}$ is most sensitive to the structural and electronic properties at the topmost surface layers. Since the strength of the contrast in Fig. 2 (b) is similar to those of Fig. 3, it is possible to state that HCI has efficiency of at least 100 times as much as that of SCI for the production of SEM contrast.

Fig. 4 shows the SEM images of $\mathrm{Si}$ irradiated with $\mathrm{Ar}^{11+} \mathrm{HCIs}$. The fluence of HCI irradiation is in the order of $10^{14} / \mathrm{cm}^{2}$. The incident electron energy for each image is $5.0 \mathrm{keV}$ for Fig. 4(a), $1.0 \mathrm{keV}$ for Fig. 4(b), and $0.5 \mathrm{keV}$ for Fig. 4(c), respectively. The SEM contrast at irradiated areas is darker than unirradiated area. This feature is contrary to that of Fig. 2. If the voltage contrast mechanism works in this case, irradiated areas became more conductive than unirradiated area, which might be caused by the potential sputtering of the native oxide layers of Si surface under the Coulomb explosion mechanism [11]. At an insulator surface the charging effect dominates the voltage contrast, and slight difference of conductivity between irradiated and unirradiated areas will raise this voltage contrast. This is also supported by the present experimental result that the contrast at unirradiated region changed brighter during SEM observation over hours, while the contrast at irradiated region did not change. The strength of the contrast between irradiated and unirradiated areas holds at higher incident electron energies. On the other hand, the contrast is weak at the incident energy of $0.5 \mathrm{keV}$. If the incident energy is decreased, the quantum efficiency of secondary electron emission approaches unity, resulting small contrast difference in SEM image. The mesh pattern was visible with an optical microscope, which means the dielectric property of the oxide layers at surface is changed by the HCI irradiation of such small fluence. 


\section{CONCLUSIONS}

We have observed secondary electron microscope (SEM) images of the surfaces of $\mathrm{Si}$ with native oxide layers and highly oriented pyrolytic graphite (HOPG) irradiated with highly charged ions (HCIs, $\mathrm{Ar}^{11+}$ ) produced by an electron beam ion source at the fluence of in the order of $10^{13} 10^{14} / \mathrm{cm}^{2}$. The SEM contrast changes brighter at irradiated areas for graphite surfaces, while the contrast for the $\mathrm{Si}$ surface become darken at irradiated regions. The results are understandable in terms of the voltage contrast mechanism. When the strength of the contrasts is roughly compared with the sample irradiated with singly charged ions (SCIs), HCI has efficiency of at least 100 times as much as that of SCI for the production of SEM contrast. The high efficiency reflects the nature of interaction between HCI and surface. The difference in the incident energy dependence of the SEM contrast also exhibits the fact that the potential energy of HCI is dissipated at topmost surface layers, while kinetic energy is distributed over many layers at surface region.

\section{REFERENCES}

[1] T. Schenkel, A.V. Hamza, A.V. Barnes, D.H. Schneider, Prog. Surface Sci. 61, 23-84 (1999).

[2] R.E. Marrs, P. Beiersdorfer and D. Schneider, Phys. Today 47(10), 27 (1994).

[3] K. Mochiji, S. Yamamoto, H. Shimizu, S. Ohtani, T. Seguchi, N. Kobayashi, J. Appl. Phys. 82, 6037-6040 (1997).

[4] M. Tona, H. Watanabe, S. Takahashi, N. Nakamura, N. Yoshiyasu, M. Sakurai, T. Terui, S. Mashiko, C. Yamada and S. Ohtani, Surface Sci. 601,723-727 (2007).

[5] M. Tona, Y. Fujita, C. Yamada and S. Ohtani, Phys. Rev. B77, 155427 (2008).

[6] T. Shinada, A. Ishikawa, M. Fujita, K. Yamashita and I. Ohdomari, Jpn. J. Appl. Phys. 38, 3419-3421 (1999).

[7] N. Yoshiyasu, S. Takahashi, M. Shibata, H. Shimizu, K. Nagata, N. Nakamura, M. Tona, M. Sakurai, C. Yamada and S. Ohtani, Jpn. J. Appl. Phys, 45, 995-997 (2006).

[8] M. Sakurai, M. Tona, S. Takahashi, H. Watanabe, N.Nakamura, N. Yoshiyasu, C. Yamada, S. Ohtani, H.A.Sakaue, Y. Kawase, K. Mitsumori, T. Terui, and S. Mashiko, Nucl. Instr. Meth. B257, 297-300 (2007).

[9] M. Sakurai, F. Nakajima, T. Fukumoto, N. Nakamura, S. Ohtani and S. Mashiko, J. Phys. Conf. Ser. 2, 52-56 (2004).
[10] M. Sakurai, H. Ohnishi, K. Asakura, M. Tona, H. Watanabe, N. Nakamura, S. Ohtani, T. Terui, S. Mashiko, H.A. Sakaue, Vacuum 84, 530-533 (2010).

[11] I.S. Bitenski, M.N. Murakhmetov, E.S. Parilis, Sov. Phys. Tech. Phys. 24, 618 (1979).

(Received September 14, 2009; Accepted February 26, 2011) 